\title{
RESEARCH
}

Open Access

\section{Akt signaling is activated by TGF $\beta 2$ and impacts tenogenic induction of mesenchymal stem cells}

Sophia K. Theodossiou, Jett B. Murray, LeeAnn A. Hold, Jeff M. Courtright, Anne M. Carper and

Nathan R. Schiele* (i)

\begin{abstract}
Background: Tissue engineered and regenerative approaches for treating tendon injuries are challenged by the limited information on the cellular signaling pathways driving tenogenic differentiation of stem cells. Members of the transforming growth factor (TGF) $\beta$ family, particularly TGF $\beta 2$, play a role in tenogenesis, which may proceed via Smad-mediated signaling. However, recent evidence suggests some aspects of tenogenesis may be independent of Smad signaling, and other pathways potentially involved in tenogenesis are understudied. Here, we examined the role of Akt/mTORC1/P70S6K signaling in early TGFß32-induced tenogenesis of mesenchymal stem cells (MSCs) and evaluated TGF $\beta 2$-induced tenogenic differentiation when Smad3 is inhibited.
\end{abstract}

Methods: Mouse MSCs were treated with TGF $\beta 2$ to induce tenogenesis, and Akt or Smad3 signaling was chemically inhibited using the Akt inhibitor, MK-2206, or the Smad3 inhibitor, SIS3. Effects of TGF 32 alone and in combination with these inhibitors on the activation of Akt signaling and its downstream targets mTOR and P70S6K were quantified using western blot analysis, and cell morphology was assessed using confocal microscopy. Levels of the tendon marker protein, tenomodulin, were also assessed.

Results: TGF $\beta 2$ alone activated Akt signaling during early tenogenic induction. Chemically inhibiting Akt prevented increases in tenomodulin and attenuated tenogenic morphology of the MSCs in response to TGF $\beta 2$. Chemically inhibiting Smad3 did not prevent tenogenesis, but appeared to accelerate it. MSCs treated with both TGF $\beta 2$ and SIS3 produced significantly higher levels of tenomodulin at 7 days and morphology appeared tenogenic, with localized cell alignment and elongation. Finally, inhibiting Smad3 did not appear to impact Akt signaling, suggesting that Akt may allow TGF 32 -induced tenogenesis to proceed during disruption of Smad3 signaling.

Conclusions: These findings show that Akt signaling plays a role in TGF $\beta 2$-induced tenogenesis and that tenogenesis of MSCS can be initiated by TGF 32 during disruption of Smad3 signaling. These findings provide new insights into the signaling pathways that regulate tenogenic induction in stem cells.

Keywords: Tendon, Tenogenesis, Mesenchymal stem cells, Tissue engineering, Akt, Smad3, TGF 32

\footnotetext{
* Correspondence: nrschiele@uidaho.edu

Chemical and Biological Engineering, University of Idaho, 875 Perimeter Dr. MS 0904, ID 83844 Moscow, USA
} 


\section{Introduction}

Tendons, the collagenous musculoskeletal tissues that connect muscle to bone to enable movement, are frequently injured and heal poorly, leading to long-term loss of function [1]. Tendon injuries are common in both the general population and athletes, and limited clinical treatment options combined with their increasing incidence and high costs in terms of both healthcare and productivity losses make tendinopathies a significant public health concern [1-3]. Mesenchymal stem cells (MSCs) are attractive for use in regenerative therapies to treat tendon injuries, as they are relatively easy to isolate and can differentiate into a variety of tissue lineages, including tendon [4-9]. However, the limited understanding of the cell signaling pathways involved in tenogenesis (differentiation toward the tendon lineage) is a challenge for tissue engineering and regenerative approaches.

Currently identified tenogenic pathways include those related to transforming growth factor beta (TGF $\beta$ ) signaling [10]. TGF $\beta$ has three isoforms (TGF $\beta 1,2$, and 3), which, in canonical signaling, appear to depend on small mothers against decapentaplegic (Smad) 2/3 signaling $[11,12]$. Smads are intracellular signaling proteins that regulate several cellular processes including growth, differentiation, and proliferation [13]. Previously, we and others showed that TGF $\beta 2$ is a potent inducer of tenogenesis in murine MSCs in vitro [9, 11, 13-19]. MSCs supplemented with recombinant human TGF 32 significantly increased production of the tendon marker proteins scleraxis and tenomodulin over 21 days (d) in culture and underwent extensive morphology changes indicative of a transition towards tendon-like cells [14]. TGF $\beta 2$ also effectively induced tenogenesis in mouse embryonic fibroblasts grown in an in vitro tendon model [13]. Interestingly, the same study showed that when Smad4, the downstream effector of Smad2 and 3, was knocked out in mouse embryonic fibroblasts via adenovirus/Cre-mediated deletion, proliferation was disrupted, but the cells still produced scleraxis, indicating early tenogenesis was able to proceed [13]. Conversely, chemical inhibition of $\mathrm{Smad} 2 / 3$ in mouse embryonic day (E) 9.5 limb buds resulted in loss of scleraxis expression in the developing tendons [11]. In a different study, following Cre-mediated deletion of $\mathrm{Smad} 2 / 3$ at postnatal day (P) 2 and 4, mutant mice did not appear to have an altered tendon phenotype when evaluated at P14 [20], suggesting loss of Smad2/3 at later developmental stages is not disruptive to tendon formation. Collectively, while canonical TGF $\beta 2$ signaling likely depends on Smad2/3, this recent evidence suggests that other cell signaling pathways may be active in tenogenesis that are independent of Smad.

Though pathways beyond TGF $\beta$ and $\operatorname{Smad} 2 / 3$ have yet to be extensively investigated in tenogenesis, the phosphatidylinositol 3 kinase (PI3K)/Akt/mTORC1/ P70S6K pathway has been implicated in the TGF $\beta$ mediated tendon response to injury [21]. Treatment of murine MSCs with TGF $\beta 1$, which induces a proinflammatory and pro-fibrogenic response in tendon [22], activated (phosphorylated) Akt [21]. Akt, a kinase with extensive downstream roles in cell survival, growth, protein synthesis, and apoptosis, is activated by the phospholipid membrane-bound PI3K in response to several extracellular cues, including various growth factors, and potentially TGF $\beta$ signaling [23]. Once phosphorylated, Akt can indirectly activate the mammalian target of rapamycin or mTORC1 complex. mTOR knockout mice display abnormalities in the gross anatomy of their Achilles, patellar, and tail tendons [21], suggesting mTOR is involved in tendon development and maintenance. mTOR also activates protein S6 kinase, or P70S6K, which mediates protein synthesis and cell growth [24]. As P70S6K is a downstream effector of Akt, it is possible that P70S6K may be activated during TGF 32 -induced tenogenesis. P70S6K has not been investigated in the context of tenogenic differentiation, though it may contribute to proliferation and migration of other fibroblastic cells, such as lung fibroblasts [25]. Overall, although components of the PI3K/Akt/ mTORC1/P70S6K pathway have been implicated in tendon development, interactions between this pathway and the tenogenic isoform of TGF $\beta$, TGF $\beta 2$, have not been investigated.

Existing data suggest activation of Akt by TGF $\beta$ signaling can take place independently of Smad2 and 3 [26], but Akt activation has not been investigated in response to TGF 32 specifically. Prior studies have mainly explored exogenous TGF $\beta 1$ to enhance TGF $\beta$ signaling in experimental settings. However, TGF $\beta$ isoforms induce differentiation towards distinct musculoskeletal tissue lineages. TGF $\beta 3$ is known to be chondrogenic [10], while TGF $\beta 1$ may induce chondrogenesis [27] or fibrosis and inflammation [28], and TGF $\beta 2$ is tenogenic [13, 14, 29, 30]. Thus, there remains a need to assess interactions between TGF $\beta 2$ and Akt signaling, specifically for tenogenesis. Notably, there is conflicting evidence as to whether Akt signaling enhances or attenuates cellular responses to TGF $\beta[31,32]$, further highlighting the need for additional studies. Though Akt signaling is a precursor to a multitude of cellular events, establishing its potential involvement in tenogenesis is beneficial due to the limited understanding of the signaling events preceding tenogenic differentiation. Non-canonical and Smadindependent interactions between TGF $\beta$ and other signaling pathways have been documented in various cell types, and summarized in a comprehensive review [24]. While TGF $\beta$ is known to interact with bone morphogenic protein (BMP), mitogen-activated protein kinase 
(MAPK), Wingless/Integrated (Wnt) [33], Hedgehog (Hh), and Notch signaling [24], potential crosstalk between TGF $\beta$ and the Akt/mTORC1/P70S6K pathway is of particular interest in the context of tenogenesis due to the availability of therapeutic agents that can target Akt/mTORC1/P70S6K [34].

Taken together, additional signaling pathways may be involved in early TGF 32 -induced tenogenesis of stem cells, and one such pathway may be PI3K/Akt/ mTORC1/P70S6K. We hypothesized that Akt/ mTORC1/P70S6K would be activated during TGF 32 induced tenogenesis, independent of Smad3 signaling. To test this, we treated mouse MSCs with TGF 32 to induce tenogenic differentiation, and chemically inhibited Akt and Smad3 signaling for up to $7 \mathrm{~d}$. We examined cell morphology, assessed activation of Akt, mTOR, and P70S6K, and evaluated levels of the tendon marker, tenomodulin. Our results showed that TGF 32 activated Akt, and inhibiting Akt prevented TGF $\beta 2$-induced tenogenesis. Inhibiting Smad3 appeared to accelerate TGF 32 -induced tenogenesis. The tenogenic involvement of pathways other than Smad3 provides additional targets for investigations in tendon development.

\section{Materials and methods}

\section{Cell culture and tenogenic induction}

Murine MSCs (C3H10T1/2, ATCC, Manassas, VA), a model MSC used in prior studies investigating tenogenesis and tendon injury [21, 29, 35], were cultured and supplemented with TGF $\beta 2$ to induce tenogenesis as previously described [14]. Briefly, cells were expanded in standard growth medium (Dulbecco's Modified Eagle's Medium (DMEM), 10\% fetal bovine serum (FBS), and 1\% Penicillin/Streptomycin) until $70 \%$ confluent and used between passages 5 and 13 . MSCs were trypsinized and seeded into each well of a 24-well plate. Cells used for 15 minutes ( $\mathrm{min}), 30 \mathrm{~min}$, 1 hour (h), and $24 \mathrm{~h}$ timepoints were seeded at 25 , 000 cells $/ \mathrm{cm}^{2}$. Cells for 3 and $7 \mathrm{~d}$ timepoints were seeded at 5000 cells $/ \mathrm{cm}^{2}$. Cells were incubated for 24 $h$ to allow for initial cell attachment, and then washed with warmed phosphate-buffered saline (PBS) (Gibco, Grand Island, NY). The medium was switched to low-serum medium (DMEM, 1\% FBS, 1\% Penicillin/Streptomycin) and allowed to equilibrate for $24 \mathrm{~h}$. Cells were rinsed with warm PBS and cultured for 15 min, $30 \mathrm{~min}, 1 \mathrm{~h}, 24 \mathrm{~h}, 3 \mathrm{~d}, 7 \mathrm{~d}$, or $14 \mathrm{~d}$ in low-serum medium with the corresponding amount of sterile water (vehicle controls) or low-serum medium supplemented with $50 \mathrm{ng} / \mathrm{mL}$ recombinant human TGF $\beta 2$ (PeproTech, Rocky Hill, NJ). The medium was changed every third day. Experiments were repeated a minimum of 3 times.

\section{Inhibition of Akt and Smad3}

To inhibit Akt signaling, cells were seeded in 24-well plates and cultured for $15 \mathrm{~min}, 30 \mathrm{~min}, 60 \mathrm{~min}, 24 \mathrm{~h}, 3 \mathrm{~d}$, and $7 \mathrm{~d}$ in low-serum medium with water and dimethyl sulfoxide (DMSO) (vehicle controls), and low-serum medium supplemented with $50 \mathrm{ng} / \mathrm{mL}$ recombinant human TGF $\beta 2$ (PeproTech), $500 \mathrm{nM}$ of the Akt inhibitor MK-2206 [36] (MedChem Express, Monmouth Junction, NJ), or both (TGF $\beta 2+$ MK-2206). To inhibit Smad3 signaling, cells were cultured and seeded into 24-well plates as described above for $15 \mathrm{~min}, 30 \mathrm{~min}, 60 \mathrm{~min}, 24 \mathrm{~h}, 3 \mathrm{~d}$, and $7 \mathrm{~d}$ in low-serum medium with water and DMSO (vehicle controls), and low-serum medium supplemented with $50 \mathrm{ng} / \mathrm{mL}$ recombinant human TGF $\beta 2$ (PeproTech), $2 \mu \mathrm{M}$ of the Smad3 inhibitor SIS3 [11] (Tocris, Bristol, UK), or both (TGF $32+$ SIS3).

\section{Western blot analysis}

Cells were collected for western blot (WB) analysis in RIPA cell lysis buffer and HALT protease inhibitor (Invitrogen, Carlsbad, CA). Sodium dodecyl sulfate (SDS) was added at a 1:1 ratio and samples were sonicated, heated to $100^{\circ} \mathrm{C}$ for $5 \mathrm{~min}$, and loaded into Novex Wedgewell 4-20\% Tris Glycine Mini Gels (Invitrogen). Lanes were loaded differentially to normalize total protein content. Cell lysate collected from each well of the 24-well plate was run in its own lane ( 2 to 3 wells of each condition were run, analyzed, and averaged per individual experiment). Samples probed for mTOR and phosphorylated (P)-mTOR required $4-12 \%$ Tris Glycine gels (Invitrogen) due to the large protein size. Following electrophoresis, gels were transferred to nitrocellulose membranes (Invitrogen), blocked in 5\% milk in Tris-buffered saline (Boston Bioproducts, Ashland, MA) with 0.1\% Tween20 (TBST) (Acros Organics, Morris Plains, NJ), and incubated overnight at $4^{\circ} \mathrm{C}$ on an orbital shaker with appropriate primary antibodies in $5 \%$ bovine serum albumin (BSA) in TBST. Primary antibodies raised in rabbit were purchased for P-Smad3, $\beta$-actin (Abcam, Cambridge MA), mTOR, P-mTOR, Akt, P-Akt, P70S6K, P-P70S6K, Smad2/3 (Cell Signaling Technologies, Danvers, MA), and tenomodulin (Tnmd) (Invitrogen) and used at concentrations of $1: 1000$ to $1: 10,000$. Phosphorylation indicates activation, and increases in levels of P-Akt, PmTOR, P-P70S6K, and P-Smad3 were used as representations of increased activation. Blots were washed $3 \times$ for $5 \mathrm{~min}$ in TBST and incubated for $1 \mathrm{~h}$ at room temperature with goat anti-rabbit HRP-linked secondary antibody (Invitrogen). Blots were then washed in TBST, developed using ECL chemiluminescence reagents (Invitrogen), imaged using a Genesis Pi6x imager (Syngene, Frederick, MD), and analyzed via band densitometry in ImageJ (NIH, Bethesda, MD), with all intensities normalized to their respective $\beta$-actin bands. 
Fluorescence staining and confocal microscopy

To observe changes in cell morphology, cells were cultured and supplemented with TGF $\beta 2$, MK-2206 and SIS3, as described above, but on glass coverslips. At 24 h, $3 \mathrm{~d}$, and $7 \mathrm{~d}$, the medium was removed, cells were rinsed with PBS, and fixed in 10\% formalin overnight at $4^{\circ} \mathrm{C}$. Cells were washed with PBS, permeabilized with $0.1 \%$ Triton-X (Acros Organics), and stained with FITCphalloidin and 4,6-Diamidino-2-phenylindole (DAPI) (Life Tech., Waltham, MA) to observe the actin cytoskeleton and cell nuclei, respectively. Coverslips were mounted on slides and imaged on a spinning-disk confocal microscope (Nikon/Andor, Melville, NY).

\section{Statistical analysis}

Proteins were initially normalized to their respective $\beta$ actin bands. As not all timepoints and experiments could be run on the same gel, treatment groups were normalized to their respective controls at each timepoint. Phosphorylated protein content was then normalized to total Akt, P70S6K, mTOR, or Smad2/3 content, as previously described [37]. Each experimental run was averaged (minimum $n=3$ independent runs, 2-3 technical replicates per run), and ratios were calculated from bands imaged on the same membrane. Resulting ratios were analyzed using 1-way analysis of variance (ANOVA) with Sidak's multiple comparison test (Prism
8, GraphPad, La Jolla, CA). Significance was set at $p<$ 0.05 . Results are reported as mean \pm standard deviation.

\section{Results}

\section{Akt signaling is activated during early TGF $\beta 2$-induced tenogenesis}

TGF $\beta 2$ treatment significantly increased the ratio of PAkt (activated) to total Akt at $30 \mathrm{~min}(p<0.05$; Fig. 1b) and $60 \mathrm{~min}(p<0.001$; Fig. 1c) and at $24 \mathrm{~h}(p<0.01$; Fig. 1d), compared to controls. TGF $\beta 2$ increased the average P-Akt/Akt ratio, though not significantly, at 15 min and $3 \mathrm{~d}$ (Fig. 1a, e). Compared to controls, TGF $\beta 2$ did not alter the ratio of P-mTOR to mTOR at any timepoint (Fig. S1A-E). TGF $\beta 2$ significantly increased the ratio of P-P70S6K to P70S6K compared to controls at $24 \mathrm{~h}(p<0.05 ;$ Fig. S2D $)$, and P-P70S6K activation trended higher at $60 \mathrm{~min}(p=0.08$; Fig. S2C). TGF $\beta 2$ did not impact the ratio of P-P70S6K to P70S6K at 15 and 30 min or 3 and $7 \mathrm{~d}$ (Fig. S2A, B, E, F).

\section{Akt inhibition disrupts early TGF $\beta 2$-induced tenogenesis}

Cells were treated with TGF $\beta 2$, MK-2206, or MK$2206+$ TGF $\beta 2$. MK-2206 effectively decreased Akt activation both on its own and when used in combination with TGF $\beta 2$. The ratio of P-Akt to Akt was significantly lower in both MK-2206 and MK-2206 + TGF $\beta 2$ treated cells, compared to TGF $\beta 2$-only treated cells at $30 \mathrm{~min}$

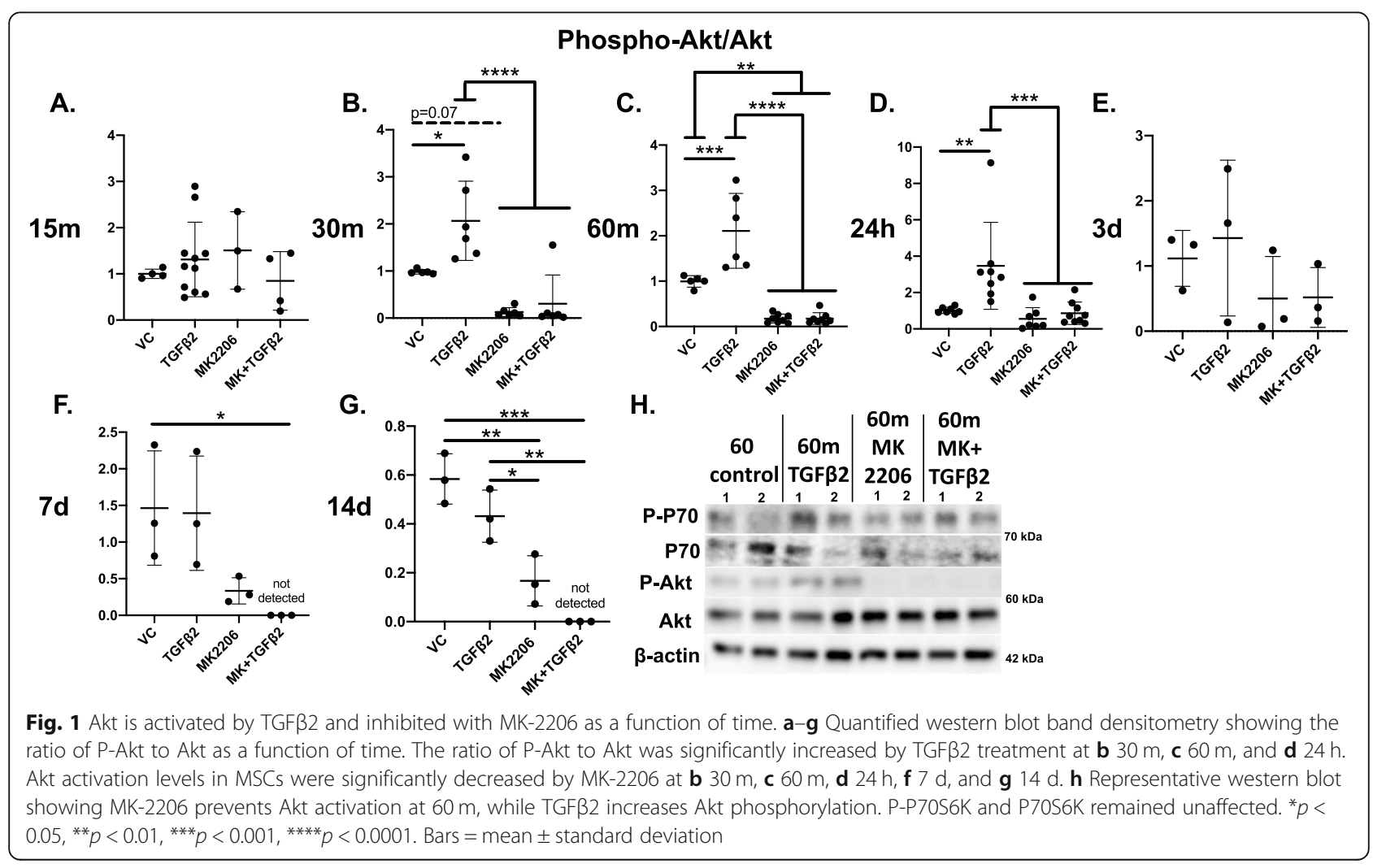


( $p<0.0001$; Fig. 1b), compared to all other groups at 60 $\min (p<0.01$ to $p<0.001$; Fig. $1 \mathrm{c}, \mathrm{h})$, and compared to TGF 32 -only treated cells $(p<0.001)$ at $24 \mathrm{~h}$ (Fig. 1d). The P-Akt to Akt ratio remained lower in MK-2206treated groups, and P-Akt was not detected in MK2206 + TGF 32 treated cells at 7 d (Fig. 1f) and $14 \mathrm{~d}$ (Fig. 1g). MK-2206 did not significantly impact the ratio of P-mTOR to mTOR at most timepoints (Fig. S1). At $24 \mathrm{~h}$, mTOR activation was significantly lower in MK2206-only treated cells, compared to TGF $\beta 2$-only cells $(p<0.05$; Fig. S1D), and at $3 \mathrm{~d}$, activation was significantly lower in MK-2206 + TGF $\beta 2$ treated cells, compared to TGF $\beta 2$-only cells $(p<0.05$; Fig. S1E). MK-2206 did not affect the ratio of P-P70S6K to P70S6K at most timepoints, though P-P70S6K activation trended lower $(p=0.08)$ in MK-2206 and MK-2206 + TGF 32 treated cells at $60 \mathrm{~min}$ (Fig. S2C) and was significantly lower $(p<0.05)$ in MK-2206 treated cells compared to TGF $32-$ only treated cells at $24 \mathrm{~h}$ (Fig. S2D). At this same timepoint, MK-2206 + TGF $\beta 2$ treated cells had a significantly higher ratio of P-P70S6K to P70S6K compared to vehicle controls $(p<0.05$; Fig. S2D), and the ratio in MK-2206+ TGF $\beta 2$ treated cells trended higher $(p=0.055)$ than in MK-2206-only treated cells. At $7 \mathrm{~d}$, levels of tenomodulin were undetectable in the MK-2206 + TGF $\beta 2$-treated cells, indicating tenogenesis had been disrupted $(p<0.01$; Fig. 2c, d). Tenogenic cell morphology was disrupted with MK-2206 + TGF $\beta 2$ treatment. Cells showed reduced elongation, proliferation, and localized alignment, compared to cells treated with TGF $\beta 2$ alone, at $24 \mathrm{~h}, 3 \mathrm{~d}$, and 7 d (Fig. 3). Finally, although cells treated with MK2206 + TGF 32 had a significantly higher ratio of P-Smad3 to $\mathrm{Smad} 2 / 3$ at $60 \mathrm{~min}(p<0.05$; Fig. S3A), levels of activated Smad3 were similar to controls by $24 \mathrm{~h}$ (Fig. S3B).

\section{Early TGF 32 -induced tenogenesis proceeds when Smad3 signaling is disrupted}

Cells were treated with TGF $\beta 2$, SIS3, or SIS3 + TGF $\beta 2$. Cells treated with SIS3 had decreased activation of Smad3 and a lower ratio $(p=0.08)$ of P-Smad3 to total Smad2/3 at $60 \mathrm{~min}$, as expected (Fig. S3C). SIS3 did not decrease the ratio of P-Akt to Akt alone or in combination with TGF $\beta 2$ at any timepoint (Fig. 4), though all conditions had significantly lower Akt activation compared to controls at $3 \mathrm{~d}$ (Fig. 4e). SIS3 did not impact the ratio of P-mTOR to mTOR, except at $15 \mathrm{~min}$, where SIS3 + TGF 32 treated cells had significantly lower mTOR activation compared to other timepoints $(p<$ 0.01 ; Fig. S4A). Finally, at $15 \mathrm{~min}$, SIS3 and SIS3 + TGF $\beta 2$ treated cells had a significantly lower ratio of P-

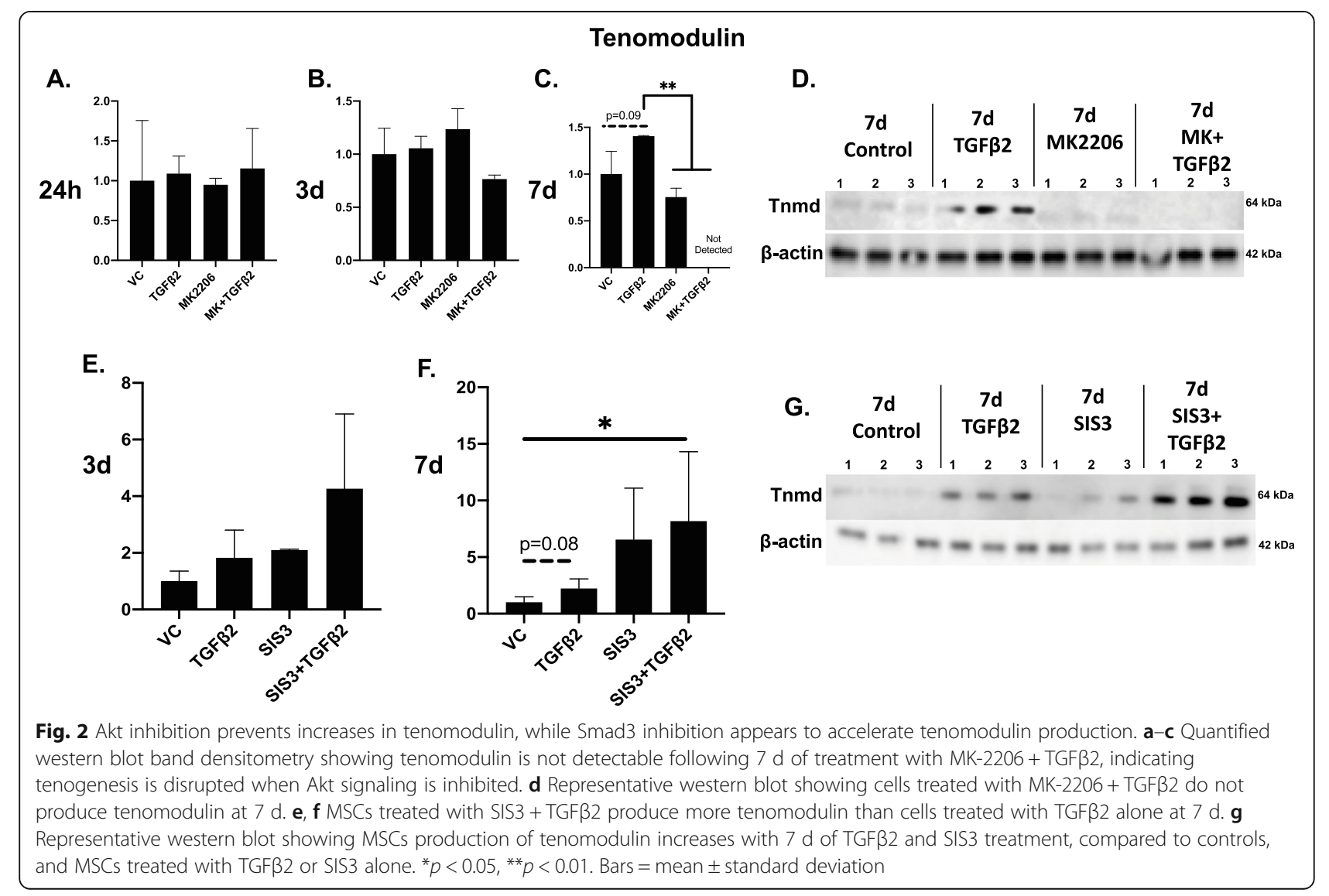




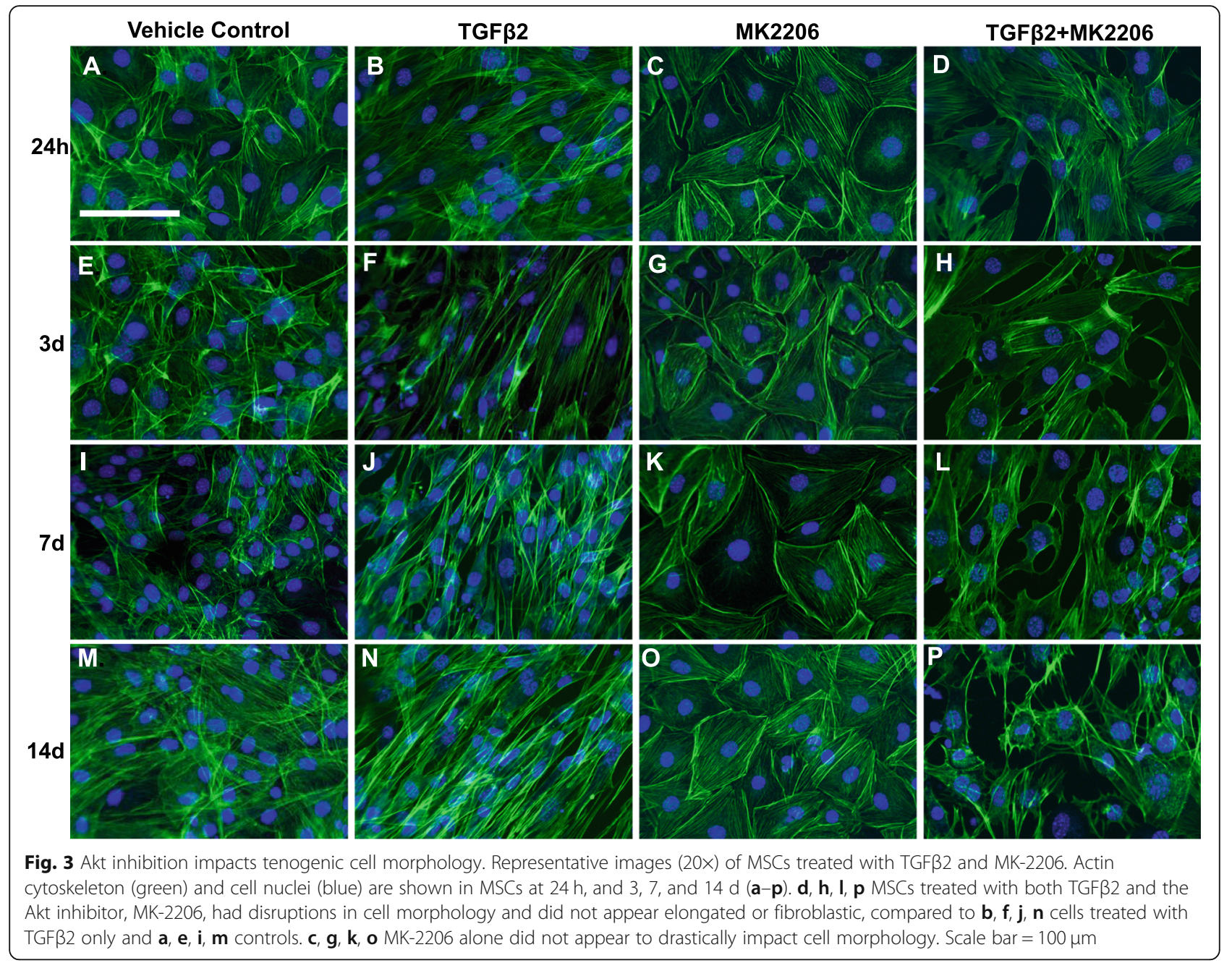

P70S6K to P70S6K $(p<0.05$; Fig. S5A), compared to cells treated with TGF $\beta 2$ alone, but SIS3 did not affect this ratio at any other timepoint (Fig. S5). Inhibiting Smad3 signaling with SIS3 did not prevent TGF $\beta 2$ induced tenogenesis. The morphology of cells treated with SIS3 + TGF $\beta 2$ displayed changes consistent with tenogenesis at earlier timepoints, compared to cells treated with TGF $\beta 2$ alone (Fig. 5). Levels of tenomodulin were also significantly higher $(p<0.01)$ at $7 \mathrm{~d}$ in cells treated with SIS3 + TGF $\beta 2$, compared to the TGF $\beta 2$ only group (Fig. 2e, f, g).

\section{Discussion}

In this study, we showed that TGF $\beta 2$ activated Akt signaling during the initiation of tenogenesis, that inhibiting Akt signaling disrupted early TGF $\beta 2$-induced tenogenesis and that TGF $\beta 2$-induced tenogenesis proceeded despite disruption of Smad3. Activation of mTOR and P70S6K, which are downstream in the Akt signaling pathway, also changed in response to TGF $\beta 2$, though these changes appeared muted compared to the impact of TGF $\beta 2$ on Akt activation. Inhibiting Akt and Smad3 had limited impact on the activation of these downstream effectors (mTOR and P70S6K). Finally, we showed that disrupting Smad3 signaling during TGF 32 induced tenogenesis appeared to accelerate morphological changes and tenomodulin production by MSCs. While previous work has shown that TGF $\beta$ signaling can proceed independently of Smad functionality [13], to our knowledge, this is the first study to investigate the activation of potential alternative cellular signaling pathways during early TGF $\beta 2$-induced tenogenesis.

Our results suggest that Akt activation is a possible tenogenic pathway that may regulate the cellular response to TGF $\beta 2$-induced tenogenesis. TGF $\beta 2$-treatment alone significantly increased Akt activation early in tenogenic induction (Fig. 1). Additionally, Akt activation with TGF $\beta 2$ across various timepoints showed an increasing trend up until $24 \mathrm{~h}$, and a decreasing trend until $14 \mathrm{~d}$, where the P-Akt to Akt ratio is significantly lower compared to $24 \mathrm{~h}$ (Fig. S6). Although the ratio of P-Akt to Akt increased by a maximum of only $\sim 2.5: 1$ in response to TGF $\beta 2$, as a kinase, Akt 


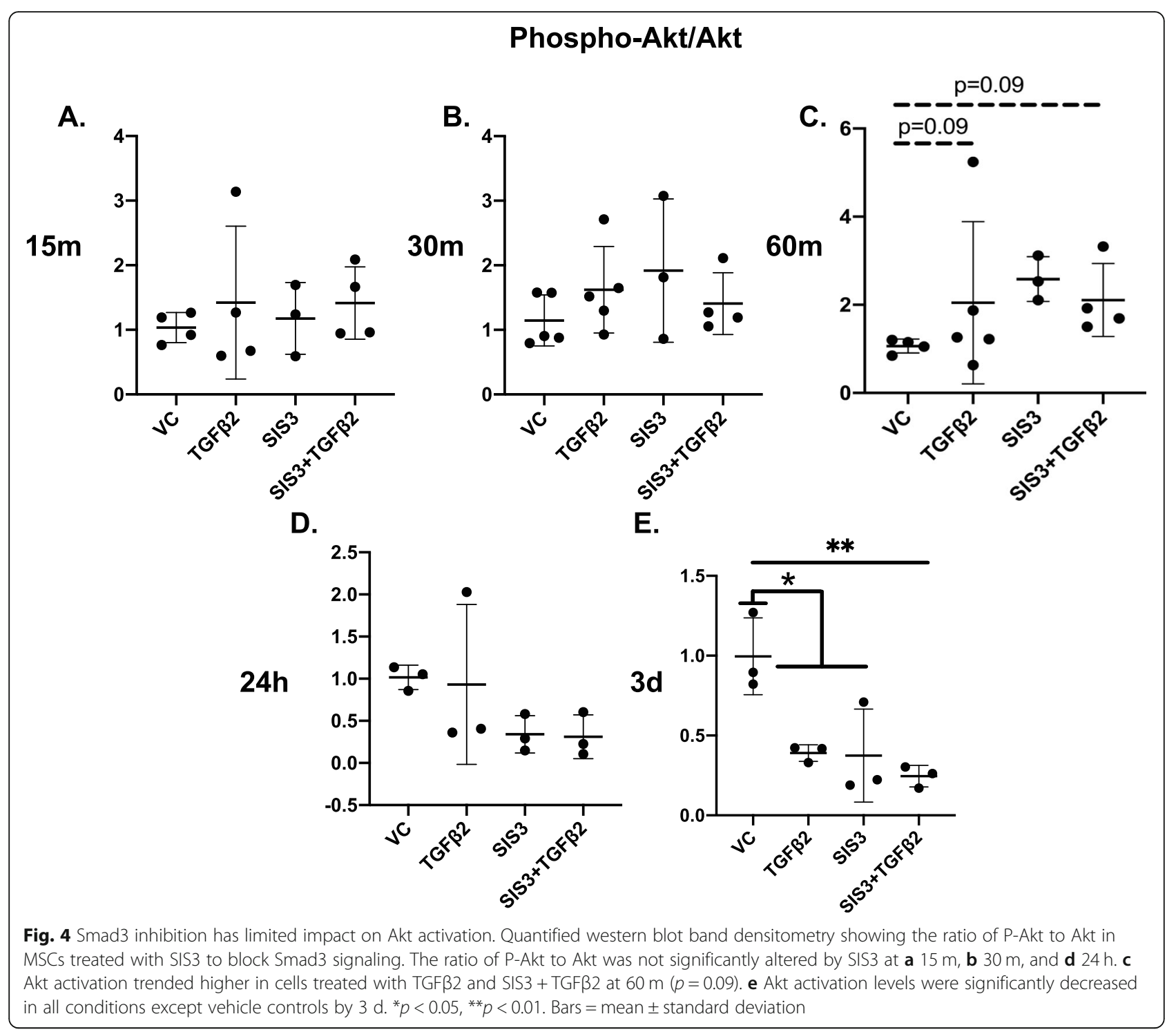

activates a cascade of intracellular events, and only modest increases in signaling may be needed to induce robust changes in transcription and protein synthesis [23]. While MK-2206 prevented Akt activation by TGF $\beta 2$ and diminished tenogenic markers, levels of total (non-phosphorylated) Akt, as well as levels of phosphorylated and nonphosphorylated mTOR and P70S6K, were not changed by MK-2206 addition alone, except at some later timepoints. This suggests that even low levels of P-Akt may be enough to maintain some downstream signaling, that other noncanonical pathways may be maintaining mTOR and P70S6K signaling in the absence of Akt activation, or that these downstream signaling molecules are not involved in TGF 32 induced tenogenesis. Overall, our findings show that TGF $\beta 2$ activates Akt signaling during early tenogenic induction.

Interestingly, in addition to not decreasing in response to MK-2206, mTOR activation was not significantly altered by TGF $\beta 2$ treatment and the apparent changes in Akt activation. This finding could be explained by Akt only indirectly activating mTOR in the canonical PI3K/ Akt/mTORC1/P70S6K pathway, and this activation may be tissue-specific $[23,38]$. The TGF $\beta$-induced epithelialto-mesenchymal transition is also regulated by mTOR [39]. Since TGF $\beta 2$-induced tenogenesis in these MSCs is occurring after the epithelial-to-mesenchymal transition that occurred in utero prior to MSC isolation, mTOR regulation may not directly influence tenogenic differentiation. It is also possible that mTOR activation is exclusive to tendon injury [21] and not activated during tenogenic differentiation. However, mTOR knockout mice display some abnormalities in gross tendon morphology [21], suggesting mTOR is involved in tendon development. Similarly, the ratio of P-P70S6K to P70S6K was not significantly increased at early timepoints. However, 


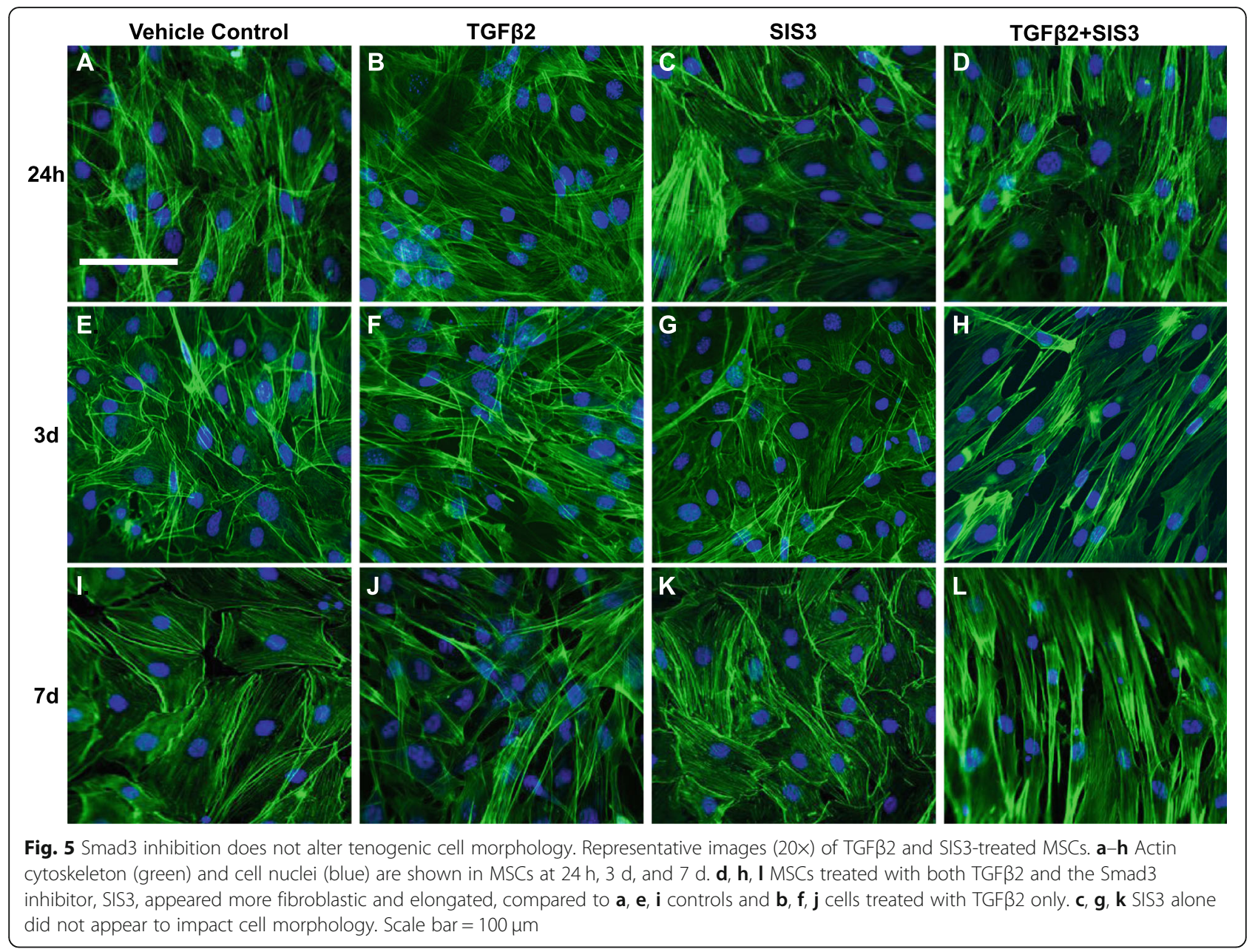

unlike mTOR, P70S6K activation was significantly higher at $24 \mathrm{~h}$ with TGF 32 treatment. The significant increases in P70S6K activation at later timepoints $(24 \mathrm{~h})$ suggest some mTOR activation, and the delayed peak in activation is logical given its position downstream in the pathway. Ultimately, both mTOR and P70S6K showed some increases (though not significant) in activation in response to TGF $\beta 2$ at earlier timepoints, indicating that the PI3K/Akt/ mTORC1/P70S6K pathway remains a potential driver of TGF 32 -induced tenogenesis.

Akt may allow TGF $\beta 2$-induced tenogenesis to proceed despite disruption of Smad3. While Smad3-independent activation of Akt by TGF $\beta$ signaling is not a novel finding [12, 26], TGF 32 -induced tenogenesis during disruption of Smad3 has not been previously shown. Inhibiting Smad3 with SIS3 did not decrease activation of Akt (Fig. 4) or downstream effectors, mTOR (Fig. S4, S5), except at isolated, later timepoints. While Akt activation did not increase significantly in the SIS3 + TGF $\beta 2$ treated cells, levels of P-Akt trended higher $(p=0.09)$ at $60 \mathrm{~m}$ (Fig. 4c), and it is possible that low levels of Akt activation are sufficient for tenogenesis to proceed. Unlike Akt, P-P70S6K activation showed an early, transient significant decrease in SIS3treated cells at $15 \mathrm{~min}$, but the ratio of P-P70S6K to P70S6K was otherwise similar to other groups at all timepoints (Fig. S5). The cause of this pattern is not clear, but could be due to effects of endogenous TGF 31 on P70S6K signaling being disrupted by SIS3 [38]. As the three isoforms of TGF $\beta$ have partially overlapping but distinct roles in musculoskeletal tissue development [40], future studies are needed to determine the specific downstream effects of each TGF $\beta$ isoform within the context of Akt signaling. Furthermore, while other pathways not assessed in this study may be initiating tenogenesis in response to TGF $\beta 2$ addition, Akt is a promising candidate due to the extensive morphological changes observed with Akt inhibition (Fig. 3). Akt inhibition does not lead to alterations in cell morphology, except in the MK-2206 + TGF $\beta 2$ groups. Similarly, SIS3 alone appears to do little to cell morphology or protein production, until combined with TGF $\beta 2$. While qualitative, the images of cell morphology showed enhanced tenogenic morphology with Smad3 inhibition and extensive disruption of tenogenic morphology with Akt inhibition. The novel finding of disrupted tenogenic morphology with 
Akt inhibition, along with loss of tenomodulin production, suggests that the Akt pathway is playing a role in early TGF 32 -induced tenogenesis.

The finding that TGF $\beta 2$ not only induces tenogenesis when Smad3 is disrupted, but that differentiation may be accelerated, is consistent with recent evidence that the downstream effector of Smad3 signaling (e.g., Smad4) may not be necessary for TGF 32 -induced tenogenic differentiation of mouse embryonic fibroblasts [13]. However, loss of Smad4 in tendon cells (via crossing Smad4 floxed mice with scleraxis-cre) resulted in joint contracture and a reduction in collagen fibril diameter, cellularity, and extracellular matrix volume in a murine model of Smad4 deletion by P5 and P14 [20]. The same study also showed that disrupting canonical TGF $\beta$ signaling using Smad2 and 3 mutant mice did not result in joint contracture when tamoxifen was administered on P2 and P4, but joint contracture was largely recapitulated by disrupting BMP signaling [20]. Taken together, our present work and these other recent studies suggest that Smad2 and 3 deletion does not disrupt TGF $\beta$ signaling in tenogenesis, but further research is needed to untangle the individual roles of Smad2 and 3, as well as the BMP-associated Smads1/5/8 [20].

Smad4 was also shown to play a role in cell proliferation in response to TGF $\beta 2$ [13]. While cellular proliferation was not quantified in this study, images show that changes in cell morphology (elongation and localized alignment) with SIS3 + TGF $\beta 2$ treatment are not accompanied by the large increases in cell number observed in prior studies of TGF 32 -only treated cells [14], suggesting Smad3 and hence downstream Smad4 signaling were effectively inhibited and may also impact proliferation. Finally, unlike TGF $\beta 2$-only and SIS3-only treated cells, most SIS3 + TGF $\beta 2$-treated groups did not survive past $10 \mathrm{~d}$ in culture, suggesting long-term SIS3 accumulation or disruption of Smad3 and downstream effectors is problematic for TGF 32 -induced tenogenesis. For this reason, only timepoints up until a maximum of $7 \mathrm{~d}$ are included in the data analysis. Overall, Smad4 appears to be necessary for maintenance of tenogenesis, but the role of Smad signaling in early tenogenesis and the dependence of TGF $\beta 2$-induced tenogenesis on Smad functionality are less clear.

It is possible that the apparent acceleration in TGF $\beta 2$ induced tenogenesis with Smad3 disruption is due to disruptions of cell signaling associated with endogenous TGF $\beta 1$. Fibroblasts and MSCs that are precursors to musculoskeletal tissues produce TGF $\beta 1$ during development $[29,30,41-43]$, and TGF $\beta 1$ signaling is known to participate in the tendon response to injury and fibrosis $[28,44-$ 46]. Multiple TGF $\beta$ isoforms may be active at any given time, but not all activate the same pathways, especially when non-canonical signaling is considered [47]. During early musculoskeletal tissue differentiation, TGF $\beta 1$ signaling may proceed exclusively via Smad, while TGF $\beta 2$ may be able to induce differentiation via alternate pathways that other TGF $\beta$ isoforms do not activate [12]. As TGF $\beta 1$ may not be tenogenic and in other studies is chondrogenic $[48,49]$ or fibrogenic [28], disrupting Smad3 may prevent the non-tenogenic effects of TGF $\beta 1$. Simultaneously, TGF $\beta 2$ signaling may proceed via an alternate pathway, thus accelerating tenogenesis when competing signals from endogenous TGF $\beta 1$ are disrupted. Other aspects of tendon development, such as production of matrix components like fibronectin and proteoglycans, as well as collagen production and organization, may require Smad4-dependent [20] and independent [50] TGF $\beta 1$ signaling, which longterm Smad3 inhibition prohibits. Furthermore, injury in a neonatal tendon is associated with $\operatorname{Smad} 2 / 3$ activation, and Smad2/3 activation meditated by TGF $\beta$ signaling through the TGF $\beta$ type I receptor ALK4/5/7 impacts regenerative healing [51]. Another Smad protein, Smad8, has also been shown to enhance regeneration of tendon injuries when MSCs are genetically engineered to overexpress both Smad8 and its downstream target, BMP2 [52, 53], suggesting that Smad signaling may be involved in tendon regeneration. Finally, interactions between multiple TGF $\beta$ isoforms and other Smads, such as Smad8 [52, 53], and downstream Smad-dependent signals, including BMPs [20, 24, 54-56], may be necessary for continued or postnatal tendon differentiation and maintenance. Interactions between Smad signaling and all three TGF $\beta$ isoforms, as well as interactions with BMP signaling, will be explored in future long-term studies. Taken together, Smad activation by TGF $\beta$ family members may play unique and time-dependent roles in differentiation and regeneration, but more work is needed to elucidate the impact of Smads in regulating tendon formation.

This study is not without its limitations. Chemical inhibitors were used to disrupt Akt and Smad3 signaling. Although western blotting showed activated Akt was lowered to almost undetectable levels by MK-2206, and the overall ratio of activated Smad3 to total Smad2/3 content decreased in SIS3-treated groups, it is possible that some activity persisted, particularly for Smad3. Future studies in animal knockouts can control for small levels of activation in proteins of interest, though other off-target effects of the deletions may confound results. For example, a mouse knockout model of almost all Akt isoforms $\left(A k t 1^{+} /^{-}, A k t 2^{-} /^{-}\right.$, and $\left.A k t 3^{-} I^{-}\right)$is viable, but the animals display generalized abnormalities in metabolism and body weight [57]. Further cellular studies in 3-dimenstional (D) constructs using knockouts are also warranted, as previous in vitro models of tenogenesis have established the need for 3D culture in evaluating long-term tenogenesis [58]. 3D studies incorporating additional tenogenic markers, such as production of collagen I and III and proteoglycans, and evaluation of 
mechanical properties, are needed. Additionally, not all proteins of interest were assessed for 7 days. Proteins activated earlier in the pathway (for example, P-mTOR) were only quantified until $24 \mathrm{~h}$ or $3 \mathrm{~d}$ in either the MK2206 + TGF $\beta 2$ or SIS3 + TGF $\beta 2$ experiments, though any notable changes in activation would likely occur within these time frames. Due to these short time frames associated with signaling activation (Akt activation was significantly increased at $30 \mathrm{~min}$ (Fig. 1b)), we assessed cell morphology and tenomodulin protein levels as markers of tenogenic induction. Our prior work showed scleraxis production in MSCs was not significantly increased until 14 and $21 \mathrm{~d}$ of TGF $\beta 2$ treatment [14]. Although some scleraxis production was detected at $7 \mathrm{~d}$ (data not shown) in the present study, the amount was not high enough to be quantified. Future studies using 3D constructs will need to assess gene expression and protein production of these tenogenic markers. We also only assessed Akt activation using a pan-Akt antibody, rather than examining individual Akt isoform activation. It is possible individual Akt isoforms impact tenogenic differentiation in different ways, though Akt isoforms are generally considered redundant, with overlapping functions in vivo [57]. Additionally, culture medium was supplemented with $50 \mathrm{ng} / \mathrm{mL}$ exogenous TGF $\beta 2$ every 3 days based on concentrations used in previous studies [11, 14, 59]. While this concentration is shown to be tenogenic in MSCs in vitro, it is possible it does not represent the in vivo availability and concentration of TGF $\beta 2$. We also did not supplement cells with other TGF $\beta$ isoforms, such as TGF $\beta 1$, or quantify TGF $\beta 1$ production by MSCs. Though TGF $\beta 1$ is generally considered fibrogenic $[21,22,28]$ and its exogenous application is used in tendon injury models, it is possible that TGF $\beta 1$ activates alternative pathways when either Akt or Smad3 signaling is disrupted. As the goal of the present study was to assess a possible signaling mechanism by which TGF $\beta 2$ initiates early tenogenesis, supplementation with other TGF $\beta$ isoforms was outside the scope of the current investigation. Future studies will integrate assessment of alternative pathways, such as those associated with other tenogenic Smads (i.e., Smad4, Smad8), BMPs, and other TGF $\beta$ isoforms. Future studies in adult human MSCs using 3D culture systems will be needed to assess the potential benefits of clinical interventions targeting these pathways. Despite various limitations, this study represents a valuable contribution to the understanding of cell signaling pathways involved in the initial stages of TGF $\beta 2$-induced tenogenesis in MSCs and can be used to inform cellular studies of early tendon development.

\section{Conclusions}

Overall, our findings show that Akt signaling is activated during TGF $\beta 2$-induced tenogenesis of MSCs, and Akt activation appears to impact tenogenic markers. Furthermore, disrupting Smad3 signaling and adding exogenous TGF $\beta 2$ did not prevent tenogenesis. Instead, tenogenic initiation appeared to be accelerated, with earlier increases in tenomodulin production and the appearance of tenogenic cell morphology. Collectively, our results suggest that Akt/mTORC1/P70S6K and other cell signaling pathways independent of Smad3 may be involved in tenogenesis. These pathways provide novel targets for future studies aiming to improve understanding of the cellular processes driving tenogenesis in stem cells.

\section{Supplementary Information}

The online version contains supplementary material available at https://doi. org/10.1186/s13287-021-02167-2.

Additional file 1: Figure S1. mTOR activation is not impacted by TGF $\beta 2$ and is decreased with Akt inhibition. Quantified western blot band densitometry showing the ratio of P-mTOR to MTOR as a measure of mTOR activation levels in MSCs. Compared to vehicle controls, the ratio of P-mTOR to mTOR was not impacted significantly by TGF $\beta 2$ treatment at any timepoint. mTOR activation levels in MSCs were significantly decreased by MK-2206 compared to TGF $\beta 2$-only treated cells at (D) $24 \mathrm{~h}$ and (E) $3 \mathrm{~d} .{ }^{*}=p<0.05$. Bars $=$ mean \pm standard deviation.

Additional file 2: Figure S2. P70S6K activation increases with TGF $\beta 2$ and is unaffected by Akt inhibition Quantified western blot band densitometry showing the ratio of P-P70S6K to P70S6K as a measure of P70S6K activation levels in MSCs. (D) The ratio of P-P70S6K to P70S6K was significantly increased by TGF 32 treatment at $24 \mathrm{~h}$, and trended higher (C) at $60 \mathrm{~m}$. P70S6K activation levels in MSCs were not significantly decreased by MK-2206 at any timepoint. ${ }^{*}=p<0.05$. Bars $=$ mean \pm standard deviation.

Additional file 3: Figure S3. TGF $\beta 2$ activates Smad3 and SIS3 decreases the ratio of P-Smad3 to Smad2/3. Quantified western blot band densitometry showing the ratio of P-Smad3 to total Smad2/3 in MSCs treated with TGF $\beta 2$ and MK-2206 or SIS3. (A) The ratio of P-Smad3 to Smad2/3 increases significantly with MK-2206 + TGF $\beta 2$ treatment at $60 \mathrm{~m}$. (B) Smad3 activation was comparable between all conditions at $24 \mathrm{~h}$. (C) The ratio of P-Smad3 to Smad2/3 trends lower ( $p=0.08$, following $60 \mathrm{~m}$ of SIS3 treatment in MSCS. ${ }^{*}=p<0.05$. Bars $=$ mean \pm standard deviation.

Additional file 4: Figure S4. mTOR activation is largely unaffected by SIS3. Quantified western blot band densitometry showing the ratio of $\mathrm{P}_{-}$ mTOR to mTOR in MSCs treated with SIS3 to block Smad3 signaling. (A) The ratio of P-mTOR to mTOR was significantly decreased by SIS3 + TGF $\beta 2$ at $15 \mathrm{~m}$, but mTOR activation was not significantly impacted at any other timepoint. ${ }^{*}=p<0.05$. Bars $=$ mean \pm standard deviation.

Additional file 5: Figure S5. P70S6K activation is largely unaffected by SIS3. Quantified western blot band densitometry showing the ratio of PP70S6K to P70S6K in MSCs treated with SIS3 to block Smad3 signaling. (A) The ratio of P-P70S6K to P70S6K was significantly decreased by SIS3, with and without TGF $\beta 2$, at $15 \mathrm{~m}$. P70S6K activation was not significantly impacted by Smad3 inhibition at any other timepoint. ${ }^{*}=p<0.05$. Bars $=$ mean \pm standard deviation.

Additional file 6: Figure S6. Akt activation as a function of tenogenic induction. Trend of the ratio of P-Akt to Akt indicates Akt activation in response to TGF $\beta 2$ peaks following $24 \mathrm{~h}$ of treatment. Akt activation then follows a decreasing trend, and is significantly lower compared to all other timepoints at $14 \mathrm{~d} .{ }^{*}=p<0.05$. Bars $=$ mean \pm standard deviation.

\section{Abbreviations}

DAPI: 4,6-Diamidino-2-phenylindole; ANOVA: Analysis of variance; BMP: Bone morphogenic protein; d: Days; DMSO: Dimethyl sulfoxide; DMEM: Dulbecco's Modified Eagle's Medium; E: Embryonic day; FBS: Fetal bovine serum; Hh: Hedgehog; mTOR: Mammalian target of rapamycin; MSCs: Mesenchymal 
stem cells; MAPK: Mitogen-activated protein kinase; P: Phospho-; PBS: Phosphate-buffered saline; PI3K: Phosphatidylinositol 3 kinase; P: Postnatal day; P70S6K: Protein S6 kinase; Smad: Small mothers against decapentaplegic; SDS: Sodium dodecyl sulfate; Tnmd: Tenomodulin; TGF $\beta$ : Transforming growth factor beta; TBST: Tris-buffered saline with $0.1 \%$ Tween20; VC: Vehicle Control; WB: Western blot; Wnt: Wingless/Integrated

\section{Authors' contributions}

SKT carried out cell culture, imaging, western blotting, and image and data analysis and drafted the manuscript. JBM carried out western blotting. LAH carried out western blotting. JMC carried out imaging and image analysis. AMC carried out imaging and image analysis. NRS conceived of the study and participated in its design and coordination and helped to draft the manuscript. All authors read and approved the final manuscript.

\section{Funding}

This work was supported by funding from the NIH/NIBIB (R03EB024134), the INBRE program, P20 GM103408 (National Institutes of General Medical Sciences), John F. Keegan Fellowship (to SKT), and Beckman Scholars Award from the Arnold and Mabel Beckman Foundation (to JBM and LAH).

\section{Availability of data and materials}

All data and materials are available upon request.

\section{Ethics approval and consent to participate}

Not applicable.

\section{Consent for publication}

Not applicable.

\section{Competing interests}

The authors have no conflicts of interest to disclose.

Received: 14 July 2020 Accepted: 14 January 2021

Published online: 26 January 2021

\section{References}

1. Thomopoulos S, Parks WC, Rifkin DB, Derwin KA. Mechanisms of tendon injury and repair. J Orthop Res. 2015;33(6):832-9.

2. Lantto I, Heikkinen J, Flinkkila T, Ohtonen P, Leppilahti J. Epidemiology of Achilles tendon ruptures: increasing incidence over a 33-year period. Scand J Med Sci Sports. 2015;25(1):e133-8.

3. Deng S, Sun Z, Zhang C, Chen G, Li J. Surgical Treatment Versus Conservative Management for Acute Achilles Tendon Rupture: A Systematic Review and Meta-Analysis of Randomized Controlled Trials. J Foot Ankle Surg. 2017;56(6):1236-43

4. Young RG, Butler DL, Weber W, Caplan Al, Gordon SL, Fink DJ. Use of mesenchymal stem cells in a collagen matrix for Achilles tendon repair. J Orthop Res. 1998;16(4):406-13.

5. Subramony SD, Dargis BR, Castillo M, Azeloglu EU, Tracey MS, Su A, Lu HH. The guidance of stem cell differentiation by substrate alignment and mechanical stimulation. Biomaterials. 2013;34(8):1942-53.

6. Kuo CK, Tuan RS. Mechanoactive tenogenic differentiation of human mesenchymal stem cells. Tissue Eng Part A. 2008;14(10):1615-27.

7. Goncalves Al, Rodrigues MT, Lee SJ, Atala A, Yoo JJ, Reis RL, Gomes ME. Understanding the role of growth factors in modulating stem cell tenogenesis. Plos One. 2013;8(12):e83734.

8. Schiele NR, Marturano JE, Kuo CK. Mechanical factors in embryonic tendon development: potential cues for stem cell tenogenesis. Curr Opin Biotechnol. 2013;24(5):834-40.

9. Brown JP, Galassi TV, Stoppato M, Schiele NR, Kuo CK. Comparative analysis of mesenchymal stem cell and embryonic tendon progenitor cell response to embryonic tendon biochemical and mechanical factors. Stem Cell Res Ther. 2015:6:89.

10. Grafe I, Alexander S, Peterson JR, Snider TN, Levi B, Lee B, Mishina Y. TGF- $\beta$ family signaling in mesenchymal differentiation. Cold Spring Harb Perspect Biol. 2018;10(5):a022202.

11. Havis E, Bonnin MA, Olivera-Martinez I, Nazaret N, Ruggiu M, Weibel J, Durand C, Guerquin MJ, Bonod-Bidaud C, Ruggiero F, Schweitzer R, Duprez D. Transcriptomic analysis of mouse limb tendon cells during development. Development. 2014;141(19):3683-96.
12. Zhang YE. Non-Smad signaling pathways of the TGF- $\beta$ family. Cold Spring Harb Perspect Biol. 2017:9:a022129.

13. Chien C, Pryce B, Tufa SF, Keene DR, Huang AH. Optimizing a 3D model system for molecular manipulation of tenogenesis. Connect Tissue Res. 2018;59(4):295-308.

14. Theodossiou SK, Tokle J, Schiele NR. TGFbeta2-induced tenogenesis impacts cadherin and connexin cell-cell junction proteins in mesenchymal stem cells. Biochem Biophys Res Commun. 2019;508(3):889-93.

15. Brown JP, Finley VG, Kuo CK. Embryonic mechanical and soluble cues regulate tendon progenitor cell gene expression as a function of developmental stage and anatomical origin. J Biomech. 2014;47(1):214-22.

16. Theodossiou SK, Murray JB, Schiele NR. Cell-cell junctions in developing and adult tendons. Tissue Barriers. 2020;8(1):1695491.

17. Lorda-Diez Cl, Montero JA, Martinez-Cue C, Garcia-Porrero JA, Hurle JM. Transforming growth factors beta coordinate cartilage and tendon differentiation in the developing limb mesenchyme. J Biol Chem. 2009; 284(43):29988-96

18. Huang AH, Lu HH, Schweitzer R. Molecular regulation of tendon cell fate during development. J Orthop Res. 2015;33:800-12.

19. Subramanian A, Kanzaki LF, Galloway JL, Schilling TF. Mechanical force regulates tendon extracellular matrix organization and tenocyte morphogenesis through TGFbeta signaling. eLife. 2018;7:e38069.

20. Schlesinger SY, Seo S, Pryce BA, Tufa SF, Keene DR, Huang AH, Schweitzer R. Loss of Smad4 in the scleraxis cell lineage results in postnatal joint contracture. Dev Biol. 2021:470:108-20.

21. Cong XX, Rao XS, Lin JX, Liu XC, Zhang GA, Gao XK, He MY, Shen WL, Fan W, Pioletti D, Zheng LL, Liu HH, Low BC, Schweitzer R, Ouyang H, Chen X, Zhou YT. Activation of AKT-mTOR signaling directs tenogenesis of mesenchymal stem cells. Stem Cells. 2018;36:527-39.

22. Maeda T, Sakabe T, Sunaga A, Sakai K, Rivera AL, Keene DR, Sasaki T, Stavnezer E, lannotti J, Schweitzer R, llic D, Baskaran H, Sakai T. Conversion of mechanical force into TGF-beta-mediated biochemical signals. Curr Biol. 2011;21(11):933-41.

23. Hers I, Vincent EE, Tavare JM. Akt signalling in health and disease. Cell Signal. 2011;23(10):1515-27.

24. Guo X, Wang XF. Signaling cross-talk between TGF- $\beta / B M P$ and other pathways. Cell Res. 2009;19(1):71-88.

25. Madala SK, Sontake V, Edukulla R, Davidson CR, Schmidt S, Hardie WD. Unique and redundant functions of p70 ribosomal 56 kinase isoforms regulate mesenchymal cell proliferation and migration in pulmonary fibrosis. Am J Respir Cell Mol Biol. 2016;55(6):792-803.

26. Wilkes MC, Mitchell H, Penheiter SG, Doré JJ, Suzuki K, Edens M, Sharma DK, Pagano RE, Leof EB. Transforming growth factor- $\beta$ activation of phosphatidylinositol 3-kinase is independent of Smad2 and Smad3 and regulates fibroblast responses via p21-activated kinase-2. Cancer Res. 2005; 65(22):10431-40

27. Tuli R, Tuli S, Nandi S, Huang X, Manner PA, Hozack WJ, Danielson KG, Hall DJ, Tuan RS. Transforming growth factor-beta-mediated chondrogenesis of human mesenchymal progenitor cells involves $\mathrm{N}$-cadherin and mitogenactivated protein kinase and Wnt signaling cross-talk. J Biol Chem. 2003; 278(42):41227-36

28. Sikes KJ, Li J, Gao S, Shen Q, Sandy JD, Plaas A, Wang VM. TGF-b1 or hypoxia enhance glucose metabolism and lactate production via HIF1A signaling in tendon cells. Connect Tissue Res. 2018;59(5):458-71.

29. Pryce BA, Watson SS, Murchison ND, Staverosky JA, Dunker N, Schweitzer R. Recruitment and maintenance of tendon progenitors by TGFbeta signaling are essential for tendon formation. Development. 2009;136(8):1351-61.

30. Tan G, Pryce BA, Stabio A, Brigande JV, Wang C, Xia Z, Tufa SF, Keene DR, Schweitzer R. TGF- $\beta$ signaling is critical for maintenance of the tendon cell fate. eLife. 2020;9:e52695

31. Asano $Y$, Ihn H, Yamane $K$, Jinnin M, Mimura $Y$, Tamaki $K$ Phosphatidylinositol 3-kinase is involved in \{alpha\}2(I) collagen gene expression in normal and scleroderma fibroblasts. J Immunol. 2004;172: 7123-35.

32. Song $K$, Wang $H$, Krebs TL, Danielpour D. Novel roles of Akt and mTOR in suppressing TGF- beta/ALK5-mediated Smad3 activation. EMBO J. 2006;25: 58-69.

33. Kishimoto $Y$, Ohkawara B, Sakai T, Ito M, Masuda A, Ishiguro N, Shukunami C. Docheva D, Ohno K. Wnt/B-catenin signaling suppresses expressions of Scx, Mkx, and Tnmd in tendonderived cells. Plos One. 2017;12(7):e0182051. 
34. Nitulescu GM, Van De Venter M, Nitulescu G, Ungurianu A, Juzenas P, Peng Q, Olaru OT, Gradinaru D, Tsatsakis A, Tsoukalas D, Spandidos DA, Margina D. The Akt pathway in oncology therapy and beyond. Int J Oncol. 2018; 53(6):2319-31.

35. Scott A, Danielson P, Abraham T, Fong G, Sampaio AV, Underhill TM. Mechanical force modulates scleraxis expression in bioartificial tendons. J Musculoskelet Neuronal Interact. 2011;11(2):124-32.

36. Hopkins BD, Pauli C, Du X, Wang DG, Li X, Wu D, Amadiume S, Goncalves MD, Hodakoski C, Lundquist MR, Bareja R, Ma Y, Harris EM, Sboner A, Beltran H, Rubin MA, Mukherjee S, Cantley LC. Suppression of insulin feedback enhances the efficacy of PI3K inhibitors. Nature. 2018;560(7719):499-503.

37. Li J, Stoppato M, Schiele NR, Graybeal KL, Nguyen PK, Kuo CK. Embryonic and postnatal tendon cells respond differently to interleukin-1 $\beta$. Ann N Y Acad Sci. 2019;1442(1):118-27.

38. Manning BD, Cantley LC. AKT/PKB signaling: navigating downstream. Cell. 2007;129(7):1261-74

39. Lamouille S, Derynck R. Cell size and invasion in TGF-\{beta\} induced epithelial to mesenchymal transition is regulated by activation of the mTOR pathway. J Cell Biol. 2007;178:437-51.

40. Xu X, Zheng L, Yuan Q, Zhen G, Crane JL, Zhou X, Cao X. Transforming growth factor- $\beta$ in stem cells and tissue homeostasis. Bone Res. 2018;6:2

41. Huang AH, Riordan TJ, Pryce BA, Weibel JL, Watson SS, Long F, Lefebvre V, Harfe BD, Stadler HS, Akiyama H, Tufa SF, Keene DR, Schweitzer R. Musculoskeletal integration at the wrist underlies the modular development of limb tendons. Development. 2015;142(14):2431-41.

42. Berthet E, Chen C, Butcher K, Schneider RA, Alliston T, Amirtharajah M. Smad3 binds Scleraxis and Mohawk and regulates tendon matrix organization. J Orthop Res. 2013;31:1475-83.

43. Clark RA, McCoy GA, Folkvord JM, McPherson JM. TGF-beta 1 stimulates cultured human fibroblasts to proliferate and produce tissue-like fibroplasia: a fibronectin matrix-dependent event. J Cell Physiol. 1997;170(1):69-80.

44. Farhat YM, Al-Maliki AA, Chen T, Juneja SC, Schwarz EM, O'Keefe RJ, Awad HA. Gene expression analysis of the pleiotropic effects of TGF- $\beta 1$ in an in vitro model of flexor tendon healing. PLoS One. 2012;7(12):e51411.

45. Juneja SC, Schwarz EM, O'Keefe RJ, Awad HA. Cellular and molecular factors in flexor tendon repair and adhesions: a histological and gene expression analysis. Connect Tissue Res. 2013;54(3):218-26.

46. Goodier HCJ, Carr AJ, Snelling SJB, Roche L, Wheway K, Watkins B, Dakin SG. Comparison of transforming growth factor beta expression in healthy and diseased human tendon. Arthritis Res Ther. 2016;18:48.

47. Bakin AV, Tomlinson AK, Bhowmick NA, Moses HL, Arteaga CL. Phosphatidylinositol 3-kinase function is required for transforming growth factor beta-mediated epithelial to mesenchymal transition and cell migration. J Biol Chem. 2000;275:36803-10.

48. Wang W, Rigueur D, Lyons KM. TGF $\beta$ signaling in cartilage development and maintenance. Birth Defects Res C Embryo Today. 2014;102(1):37-51.

49. Xia P, Wang X, Qu Y, Lin Q, Cheng K, Gao M, Ren S, Zhang T, Li X. TGF- $\beta 1$ induced chondrogenesis of bone marrow mesenchymal stem cells is promoted by low-intensity pulsed ultrasound through the integrin-mTOR signaling pathway. Stem Cell Res Ther. 2017;8:281.

50. Hocevar BA, Brown TL, Howe PH. TGF- $\beta$ induces fibronectin synthesis through a c-Jun N-terminal kinase-dependent, Smad4-independent pathway. EMBO J. 1999;18(5):1345-56.

51. Kaji DA, Howell KL, Balic Z, Hubmacher D, Huang AH. Tgf $\beta$ signaling is required for tenocyte recruitment and functional neonatal tendon regeneration. eLife. 2020;9:e51779.

52. Hoffmann A, Pelled G, Turgeman G, Eberle $P$, Zilberman $Y$, Shinar $H$, KeinanAdamsky K, Winkel A, Shahab S, Navon G, Gross G, Gazit D. Neotendon formation induced by manipulation of the Smad8 signalling pathway in mesenchymal stem cells. J Clin Investig. 2006;116(4):940-52.

53. Pelled G, Snedeker JG, Ben-Arav A, Rigozzi S, Zilberman Y, Kimelman-Bleich N, Gazit Z, Muller R, Gazit D. Smad8/BMP2-engineered mesenchymal stem cells induce accelerated recovery of the biomechanical properties of the Achilles tendon. J Orthop Res. 2012;30(12):1932-9.

54. Lorda-Diez CI, Montero JA, Choe S, Garcia-Porrero JA, Hurle JM. Ligand- and stage-dependent divergent functions of BMP signaling in the differentiation of embryonic skeletogenic progenitors in vitro. J Bone Miner Res. 2014; 29(3):735-48.

55. Shen H, Gelberman RH, Silva MJ, Sakiyama-Elbert SE, Thomopoulos S. BMP12 induces tenogenic differentiation of adipose-derived stromal cells. Plos One. 2013;8(10):e77613.
56. Lee JY, Zhou Z, Taub PJ, Ramcharan M, Li Y, Akinbiyi T, Maharam ER, Leong DJ, Laudier DM, Ruike T, Torina PJ, Zaidi M, Majeska RJ, Schaffler MB, Flatow EL, Sun HB. BMP-12 treatment of adult mesenchymal stem cells in vitro augments tendon-like tissue formation and defect repair in vivo. Plos One. 2011;6(3):e17531.

57. Dummler B, Tschopp O, Hynx D, Yang Z, Dirnhofer S, Hemmings BA. Life with a single isoform of Akt: mice lacking Akt2 and Akt3 are viable but display impaired glucose homeostasis and growth deficiencies. Mol Cell Biol. 2006;26(21):8042-51.

58. Butler DL, Juncosa-Melvin N, Boivin GP, Galloway MT, Shearn JT, Gooch C, Awad $\mathrm{H}$. Functional tissue engineering for tendon repair: a multidisciplinary strategy using mesenchymal stem cells, bioscaffolds, and mechanical stimulation. J Orthop Res. 2008;26(1):1-9.

59. Havis E, Bonnin MA, de Lima JE, Charvet B, Milet C, Duprez D. TGF $\beta$ and FGF promote tendon progenitor fate and act downstream of muscle contraction to regulate tendon differentiation during chick limb development. Development. 2016;143:3839-51.

\section{Publisher's Note}

Springer Nature remains neutral with regard to jurisdictional claims in published maps and institutional affiliations.
Ready to submit your research? Choose BMC and benefit from:

- fast, convenient online submission

- thorough peer review by experienced researchers in your field

- rapid publication on acceptance

- support for research data, including large and complex data types

- gold Open Access which fosters wider collaboration and increased citations

- maximum visibility for your research: over $100 \mathrm{M}$ website views per year

At BMC, research is always in progress.

Learn more biomedcentral.com/submissions 